\title{
Zukunftstechnologien für aktives Altern
}

\section{DIE ALTERNDE GESELLSCHAFT - MÖGLICHKEITEN UND HERAUSFORDERUNGEN}

Derzeit vollzieht sich überall auf der Welt ein einschneidender demografischer Wandel: Noch niemals zuvor ist die Bevölkerung in fast allen Industrie- und Entwicklungsländern so stark gealtert. Einige Experten sprechen gar von einer demografischen Revolution, die alle Kulturen und Gesellschaften erfasst. Tatsächlich lässt sich das Phänomen der alternden Gesellschaft bereits seit einigen Jahren in hoch industrialisierten Ländern wie Japan, Italien oder Deutschland beobachten. Weil das Bild des Alltags dort zunehmend von alten Menschen geprägt wird, sind sich Angehörige aller Altersgruppen inzwischen eher bewusst, dass sie in einer Mehr-Generationen-Gesellschaft leben und nicht in einer der ewigen Jugend - wie es viele Zeitschriften, die Werbung und das MainstreamKino suggerieren. Tatsächlich hat die Alterung der Bevölkerung einen wachsenden Einfluss auf die Strukturen der globalen Arbeits- und Kapitalmärkte sowie auf die sozialen Dienstleistungs- und Sicherungssysteme, wie Gesundheitsversorgung und die Renten. Vor diesem Hintergrund können weder Gesellschaft noch Politik das Alterungsphänomen einfach ignorieren und zwar ganz unabhängig davon, ob darin eine positive oder negative Entwicklung gesehen wird.

In der öffentlichen Diskussion wird die demografische Herausforderung zumeist als ernsthafte Bedrohung der sozialen Sicherungssysteme dargestellt und im Allgemeinen in negativer Art und Weise diskutiert (Stichwort „Kostenexplosion“). Eine andere Sichtweise eröffnet sich, wenn das Potenzial alternder Gesellschaften diskutiert wird: zum Beispiel die Schaffung neuer Märkte für innovative Anwendungen, neue Produkte und Dienstleitungen, die auf die Bedürfnisse älterer Menschen zugeschnitten sind. ${ }^{1}$ Beide Betrachtungsweisen haben ihre Berechtigung und sollten im Zusammenhang miteinander und im Hinblick darauf diskutiert werden, wie wir uns auf die Auswirkungen des demografischen Wandels vorbereiten und Einfluss auf die
Gestaltung der alternden Gesellschaft nehmen können anstatt in dem Glauben zu verharren, dieser demografische Wandel käme wie eine Naturkatastrophe über uns und treffe direkt den Kern unserer Zivilisation.

Die folgenden Ausführungen sollen dazu anregen, die Forschung wie auch Debatten über politische Optionen zur Gestaltung des demografischen Wandels zu aktivieren. Ich konzentriere mich auf Anwendungen der Informations- und Kommunikationstechnologie (IuK), da hier bereits jetzt einige vielversprechende Lösungen für den Bereich aktives Altern angeboten werden. ${ }^{2}$ Die zentrale Frage lautet, wie den Herausforderungen begegnet und wie das Potenzial einer alternden Gesellschaft genutzt werden kann, und zwar aus dem Blickwinkel des Forschungsansatzes „Zukunftstechnologien für aktives Altern“ (Future Technologies for Active Aging (FT4AA)). ${ }^{3}$

\section{POLITISCHE PROGRAMME FÜR AKTIVES ALTERN}

Die Weltgesundheitsorganisation (WHO) definiert den im aktuellen Politikdiskurs zunehmend verwendeten Begriff „aktives Altern" folgendermaßen:

„Unter aktivem Altern versteht man den Prozess der Optimierung der Möglichkeiten von Menschen, im zunehmenden Alter ihre Gesundheit zu wahren, am Leben ihrer sozialen Umgebung teilzunehmen und ihre persönliche Sicherheit zu gewährleisten und derart ihre Lebensqualität zu verbessern. Der Ausdruck „aktives Altern” kann sowohl auf Einzelpersonen als auch auf ganze Bevölkerungsgruppen bezogen werden. Aktives Altern ermöglicht den Menschen, ihr Potenzial für körperliches, soziales und geistiges Wohlbefinden im Verlaufe ihres gesamten Lebens auszuschöpfen und am sozialen Leben in Übereinstimmung mit ihren Bedürfnissen, Wünschen und Fähigkeiten teilzunehmen; gleichzeitig soll für Hilfsbedürftige ausreichender Schutz, Sicherheit und Pflege gewährleistet sein.“4 Das Wort „aktiv” bezieht sich auf die andauernde Teilhabe am sozialen, wirt- schaftlichen, kulturellen, spirituellen und zivilen Leben, also nicht allein auf die Möglichkeit, körperlich aktiv oder erwerbstätig zu sein. Hinsichtlich der Erwerbstätigkeit schlägt die WHO vor, nicht nur auf den formalen Arbeitsmarkt zu schauen, sondern auch zu bedenken, dass ältere Menschen häufig im informalen Sektor tätig sind und darüber hinaus unbezahlte Arbeit zuhause oder in ihren Familien leisten.

Das Konzept des „aktiven Alterns“, das sowohl die Probleme als auch die Möglichkeiten des demografischen Wandels umfasst, ist Bestandteil des politischen Diskurses in Europa, Gleiches gilt für das Bestreben, relevante Akteure eng in den politischen Prozess einzubinden. Die Europäische Union (EU) fördert gezielt politische Maßnahmen für aktives bzw. wohltuendes Altern wie auch deren Umsetzung, beispielsweise durch Aktionsprogramme für lebenslanges Lernen oder für gesundheitsschonende Arbeitsabläufe. Sie unterstützt eine längere Erwerbstätigkeit, einen

Gassmann, O./Reepmeyer, G. (2006): Wachstumsmarkt Alter, München und Wien.

2 Der Inhalt dieses Artikels basiert auf Arbeiten, die der Autor als Senior-Gastwissenschaftler der Europäischen Kommission, DG Joint Research Centre (JRC) Institute for Prospective Technological Studies (IPTS), Abteilung Informationsgesellschaft in Sevilla im Zeitraum 2005-07 durchgeführt hat. Für den Inhalt dieses Artikels ist der Autor verantwortlich. Die geäußerten Auffassungen stimmen nicht unbedingt mit der Meinung der Europäischen Kommission überein.

3 Die Debatte über Zukunftstechnologien verstehe ich als komplementär zu anderen Konzepten aktiven Alterns, wie z. B. "gesunde Ernährung" oder "Sport und Gesundheit".

$4 \quad$ Weltgesundheitsorganisation (2002): Aktiv Altern. Rahmenbedingungen und Vorschläge für politisches Handeln, Genf, S. 12.

Norbert Malanowski, Dr., VDI Technologiezentrum $\mathrm{GmbH}$, Düsseldorf, arbeitet zu den ökonomischen und sozialen Aspekten zukünftiger Technologien für alternde Gesellschaften und berät die Europäische Kommission und das Bundesministerium für Bildung und Forschung.

e-mail: malanowski@vdi.de 
schrittweisen Rückzug aus dem Arbeitsleben, ein aktives Leben auch während der Rente sowie die Teilnahme an gesundheitsfördernden Aktivitäten.

\section{DER „SILBERNE“ MARKT}

Derzeit wird in Zeitungen und Zeitschriften lebhaft über das wirtschaftliche Potenzial des „silbernen“ Marktes debattiert. Gemeint ist damit das Marktpotenzial von Produkten und Dienstleistungen, die speziell auf die Bedürfnisse älterer Menschen zugeschnittenen sind. In der Regel wird suggeriert, es genüge, wenn Unternehmen das passende "technologische" Angebot (also Anwendungen, Produkte und Dienstleistungen) für diese Zielgruppe entwickeln würden, um dann von der starken Kaufkraft der älteren Bevölkerung zu profitieren. Tatsächlich aber ist es gar nicht so einfach, die Bedürfnisse älterer Menschen nach angepassten Technologien tatsächlich auch in eine starke Nachfrage nach altengerechten Produkten umzusetzen und damit Gewinn aus dem demografischen Wandel zu ziehen. Unternehmen wie Microsoft, Hewlett Packard und Intel sind dahingehend initiativ geworden, dass bei Neuentwicklungen oder Neuauflagen existierender IuK-Produkte auch die besonderen Anforderungen berücksichtigt werden, die ältere Menschen an technologische Produkte stellen. Ob derartige Initiativen eher singulär sind oder eine Streuwirkung innerhalb der US-Wirtschaft haben, ist nicht bekannt. Für die Schweiz gibt es entsprechende Erkenntnisse: Demnach hält es zwar die überwiegende Mehrheit der Schweizer Firmen für wichtig, sich im Rahmen ihrer Produktpolitik an den demografischen Wandel anzupassen. Gleichwohl hat nur ein Drittel der Firmen eine Vorstellung davon, wie altengerechte Produkte angeboten und vermarktet werden können. ${ }^{5}$ Vermutlich sind es gerade die kleinen Unternehmen, die den Schritt hin zu Produktinnovationen noch nicht gewagt haben, weil ihnen die notwendigen Ressourcen fehlen, um genaue Informationen über den „silbernen Markt" einzuholen. Offenbar haben wir es hier mit einem „Teufelskreis“ zu tun: Die älteren Konsumenten (und der Markt) warten auf altengerechte Produkte (Angebot), und die Unternehmen warten ihrerseits auf Signale des Marktes (Nachfrage).

Die zitierte Studie macht auf zwei wichtige Aspekte aufmerksam: Zum einen kann für das Nachfrageverhalten entscheidend sein, dass Veränderungen bei Anwendungen, Produkten und Dienstleistungen überhaupt wahrgenommen werden bzw. wie sie wahrgenommen werden. Zum anderen müssen die neuen Anwendungen und Produkte erschwinglich sein. Auch eine noch so effektive seniorengerechte Anwendung, ein noch so gutes Produkt, nützt so gut wie nichts, wenn es sich kaum jemand leisten kann. Diese Aspekte sollten bedacht werden, wenn es darum geht, die relevanten Akteure auf intelligente Weise einzubinden.

\section{DIE EINBINDUNG DER GESELLSCHAFT- LICHEN INTERESSENGRUPPEN}

In wissenschaftlichen und politischen Debatten, aber auch in der Industrie und bei den meisten Interessenvertretungen älterer Menschen, gibt es einen Konsens, dass die Beteiligung älterer Menschen an der Entwicklung zukünftiger Anwendungen, Produkte und Dienstleistungen nicht nur für die Senioren selbst relevant sei, sondern für die Gesellschaft als Ganzes. „Partizipation“ erscheint als zielführender Weg, um den mit den alternden Gesellschaften verbundenen Herausforderungen zu begegnen und die sich bietenden Chancen zu nutzen. In politischen Konzepten, wie beispielsweise denen der WHO zu „Aktiv Altern“ oder auch in der ministeriellen Erklärung der Europäischen Kommission zum Thema „IuK-Technologien für eine integrative Gesellschaft", ist die Beteiligung der älteren Bevölkerung und ihrer Interessenvertretungen mit dem Ziel, Angebot und Nachfrage zusammenzubringen, eines der tragenden Elemente. ${ }^{6}$ Die damit verbundene Wahrnehmung der älteren Generation als "grey power" hält der britische Sozialwissenschaftler Alan Walker allerdings eher für eine modische Interpretation, die keine solide Basis habe. Interessengruppen älterer Menschen könnten sich meist nur sehr kleine Geschäftsstellen leisten und hätten aufgrund mangelnder finanzieller Mittel schwer zu kämpfen. Zudem gebe es eine große Anzahl älterer Menschen, die ihre Partner, Angehörigen oder andere Menschen pflegten. Viele hätten daher weder die körperliche Kraft noch die mentale Energie, sich darüber hinaus in partizipatorischen Prozessen zu engagieren. ${ }^{7}$

Doch auch jenseits der Frage, welche Bedeutung man partizipatorischen Ansätzen zuspricht, bleibt die Notwendigkeit, ein weiter ausdifferenziertes Wissen über die spezifischen technologiebezogenen Bedürfnisse von unterschiedlichen Gruppen älterer Menschen zusammenzutragen. Nur so werden Anwendungen, Produkte und Dienstleistungen auf den Markt kommen, die ihren ,tatsächlichen “ Bedürfnissen entsprechen.

\section{ZUKUNFTSTECHNOLOGIEN FÜR AKTIVES ALTERN}

Was bedeutet das Vorgesagte für einen neuen Forschungsansatz ,Zukunftstechnologien für aktives Altern“ (FT4AA), der Anwendungen von IuK-Technologien in den Mittelpunkt stellt? Um Missverständnissen vorzubeugen: Wenn wir von technologischen Innovationen sprechen, geht es nicht um den Versuch, Alterungsprozesse umkehren zu wollen. Der Einsatz von $\mathrm{Zu}-$ kunftstechnologien für eine Politik des aktiven Alterns zielt vielmehr darauf ab, erstens, signifikante physische und kognitive Einschränkungen möglichst zu vermeiden, zweitens, die von älteren Menschen wahrgenommene Lebensqualität zu verbessern, drittens, das Erfahrungswissen und die Kenntnisse älterer Menschen so lange wie möglich zu nutzen und schließlich, viertens, die Ausgaben der öffentlichen Haushalte zu stabilisieren. Darüber hinaus kann es von Fall zu Fall auch notwendig sein, ethische Fragen zu diskutieren (z. B. hinsichtlich der Unabhängigkeit älterer Menschen). Der Ansatz des aktiven Alterns berücksichtigt auch die „Komplexität des Alterns". Anstatt einfach die herkömmliche Aufteilung in Alterskategorien vorzunehmen (die Gruppe der 55 bis 64-Jährigen, der 65 bis 74-Jährigen und der über 75-Jährigen) wird bei diesem Ansatz eine wesentlich differenziertere Kategorisierung von Alter zugrunde gelegt:

- Alter liegt (mehr oder weniger) nahe am Rentenalter (A: guter gesundheitlicher $\mathrm{Zu}-$ stand; B: starke gesundheitliche Beeinträchtigungen);

\footnotetext{
5 Vgl. Gassmann/Reepmeyer, a.a. O.

6 European Commission (2006): ICT for an Inclusive Society, Ministerial Declaration of the Ministerial Conference in Riga, Brussels.

7 Walker, A. (2006): Aging and Politics - An International Perspective, in: Binstock, R./George, L. K. (Hrsg.): Handbook of Aging and the Social Sciences, Elsevier Academic Press, Amsterdam u.a., S. 339-359.
} 


\section{Abb. 1: Risiken bei Produktinnovationen und technologiebezogene Bedürfnisse älterer Menschen}

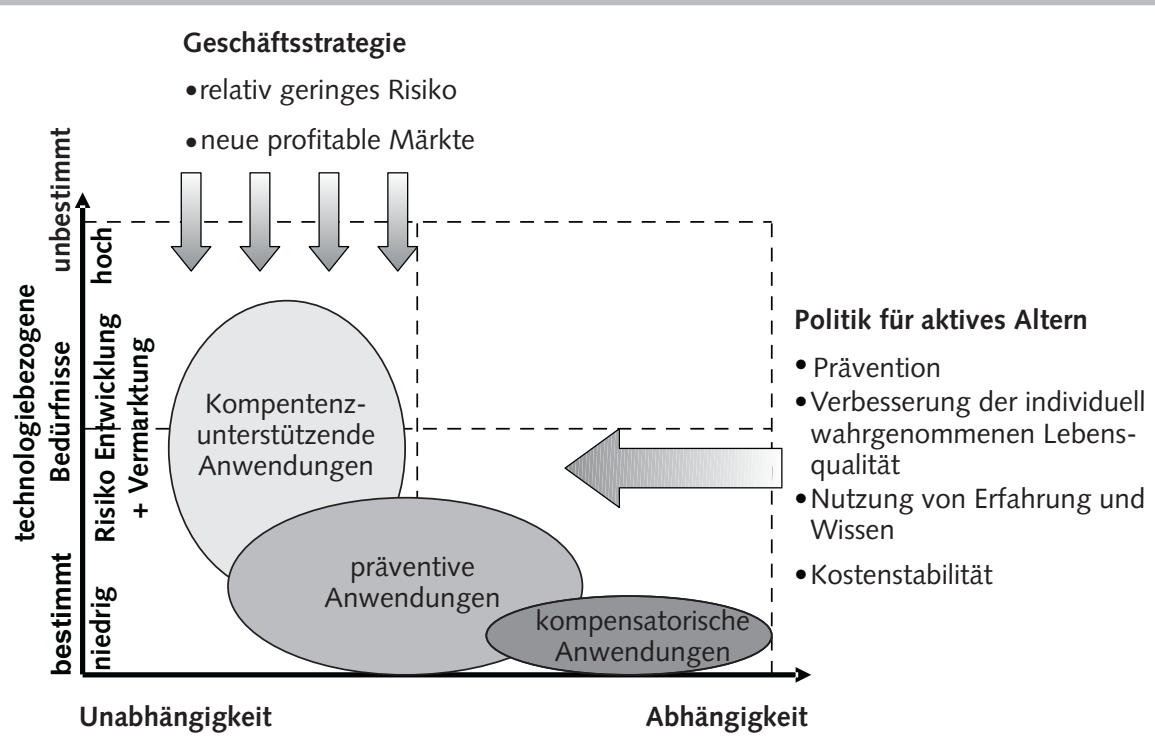

Quelle: Darstellung des Autors.

WSI MITEELUNGEN

- Autonomes Alter als Rentner (Phase der unabhängigen Lebensführung);

- Alter mit wachsenden Beeinträchtigungen (Beginn der Phase der abhängigen Lebensführung);

- Alter der abhängigen Lebensführung als Rentner.

Weitere wichtige Elemente des FT4AAAnsatzes sind

- das Prinzip der Generationengerechtigkeit. Damit werden der Lastenausgleich und die Solidarität zwischen den Generationen in alternden Gesellschaften betont, damit es aufgrund gestiegener Ausgaben der öffentlichen Haushalte für Ältere nicht zu Verteilungskonflikten zwischen den Generationen kommt;

- die spezifischen technologiebezogenen Bedürfnisse älterer Menschen. Um diese Bedürfnisse älterer Menschen (Nachfrage) mit dem "technologischen“ Angebot (an Anwendungen, Produkten und Dienstleistungen) in Einklang zu bringen, werden besondere "Werkzeuge“ gebraucht (ein Beispiel dafür ist Design for All);

- die Unterscheidung zwischen Anwendungen kompensatorischer, präventiver und kompetenzunterstützender Art;

- die Suche nach passenden Kommunikationsmethoden und -werkzeugen, um Brücken zwischen den verschiedenen relevanten Interessengruppen zu schlagen;
- nachhaltige Kommunikationsplattformen für die Interessengruppen (z. B. von älteren Menschen, der Industrie, der Wissenschaft, von Regierungsorganisationen und anderen Organisationen wie Gewerkschaften)

Als Rahmenbedingung wird die politische Unterstützung für die Entwicklung altengerechter Produkte und Dienstleistungen notwendig sein. Um das Risiko zu minimieren, bei Produktneuentwicklungen am Bedarf vorbei zu produzieren, müssen Unternehmen, die altengerechte Anwendungen, Produkte und Dienstleistungen in $\mathrm{Zu}$ kunft produzieren wollen, mehr Wissen über die spezifischen technologiebezogenen Bedürfnisse der älteren Menschen einholen. Das Risiko einer Neuentwicklung und das Risiko, am Markt vorbei zu produzieren, schwankt zwischen hoch und niedrig. Die Art des technologiebezogenen Bedarfs bei älteren Menschen pendelt zwischen bestimmt und unbestimmt. Beide Funktionen hängen aber unmittelbar miteinander zusammen (Abbildung 1): Wenn die technologiebezogenen Bedürfnisse diffus sind, ist das Risiko für die Entwicklung und Vermarktung einer Produktinnovation groß. Umgekehrt gilt gleichermaßen, dass das Risiko für eine Produktinnovation gering ist, wenn es einen konkreten technologiebezogenen Bedarf gibt. Im Moment führt dieses Abhängigkeitsverhältnis dazu, dass die meisten der angebotenen Produk- te bzw. Dienstleistungen im Bereich der kompensatorischen Maßnahmen liegen und somit der Großteil wirtschaftlicher und gesellschaftlicher Ressourcen eingesetzt wird, um signifikante physische oder kognitive Einschränkungen im Leben älterer Menschen auszugleichen. Ziel der Anwendung des FT4AA-Ansatzes (Abbildung 1) ist es nun, die Notwendigkeit kompensatorischer Maßnahmen zu reduzieren, sodass sowohl der Einsatz präventiver als auch kompetenzunterstützender Maßnahmen deutlich zunehmen wird. Anwendungen im Bereich der Prävention sind nämlich darauf ausgerichtet, bedeutende physische und kognitive Einschränkungen bereits im Vorfeld zu vermeiden. Präventivanwendungen sollen den Einsatz kompensatorischer Maßnahmen möglichst weit hinausschieben. Kompetenzunterstützende Anwendungen gehen sogar noch einen Schritt weiter. Während sich kompensatorische und präventive Anwendungen grundsätzlich auf vorhandene Einschränkungen oder Behinderungen richten, besteht das Prinzip kompetenzunterstützender Anwendungen darin, ältere Menschen in ihren speziellen Fähigkeiten gezielt zu fördern.

Die mit Produktinnovationen verbundenen Risiken bei Entwicklung und Marketing können dadurch vermindert werden, dass mit Hilfe der oben angesprochenen Kommunikationsprozesse die technologiebezogenen Wünsche und Bedürfnisse älterer Menschen ermittelbar sind, sodass Angebot und Nachfrage aufeinander abgestimmt werden können.

\section{FAZIT}

Die Entwicklung und Nutzung von $\mathrm{Zu}-$ kunftstechnologien für das aktive Altern sind ein zukunftsweisender Ansatz, um sich den Herausforderungen alternder Gesellschaften zu stellen und zugleich möglichst viele der sich bietenden Potenziale in intelligenter Weise auszuschöpfen. Der Prozess alternder Gesellschaften findet nicht allein in Industrieländern statt. Vielmehr kann man von einem allgemeinen Trend sprechen, der weltweit zu beobachten ist, auch wenn sich die demografische Entwicklung in einzelnen Ländern unterschiedlich schnell vollzieht. Die EU und ihre Mitgliedsstaaten können eine Vorreiterrolle übernehmen, indem sie das Konzept „Zukunftstechnologien für aktives Altern“ vorantreiben und zwar nicht als Substitut für andere Konzepte (wie Gesundheits- 
und Ernährungsprogramme), sondern in Ergänzung zu ihnen. Länder, die sich dafür engagieren, Zukunftstechnologien im weitesten Sinne und IuK-Anwendungen im Besonderen in den Dienst des aktiven Al- terns zu stellen, können sich einen Wettbewerbsvorteil sichern. Ein wirtschaftlich und gesellschaftlich günstiges Umfeld für Forschung und Entwicklung sind der Boden, auf dem diese Innovationen und Ver- besserungen von Anwendungen, Produkten und Dienstleistungen in Europa gedeihen können. 